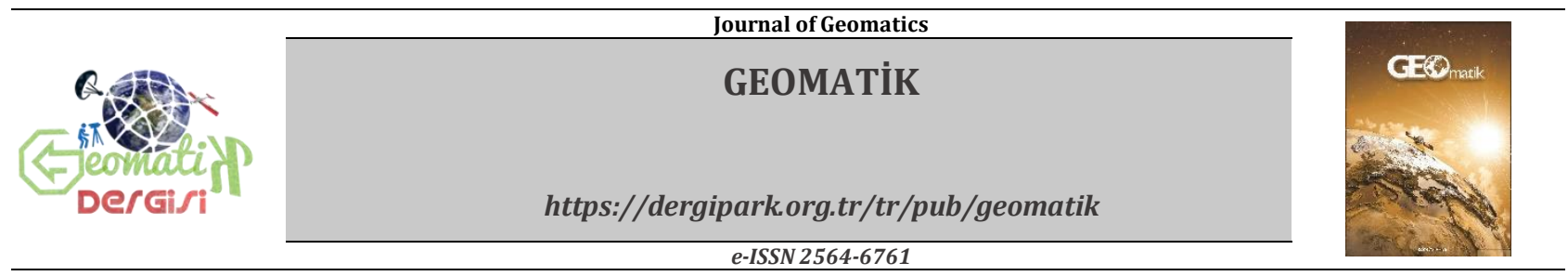

\title{
Web tabanlı GNSS Yazılımlarının (CSRS-PPP, Trimble-RTX) Performansının Araştırılması
}

\author{
Samet İnyurt*1, Mustafa Ulukavak² \\ ${ }^{1}$ Bülent Ecevit Üniversitesi, Mühendislik Fakültesi, Geomatik Mühendisliği Bölümü, Zonguldak, Türkiye \\ ${ }^{2}$ Harran Üniversitesi, Mühendislik Fakültesi, Harita Mühendisliği Bölümü, Şanlıurfa, Türkiye
}

\author{
Anahtar Kelimeler \\ GNSS \\ CSRS-PPP \\ Trimble-RTX
}

\begin{abstract}
ÖZ
Son yıllarda internet üzerinden çevrim içi GNSS değerlendirme servisleri konum elde etmede önemli bir araç olarak kullanılmaktadır. Bu sistemlerin en önemli özelliği GNSS verilerinin değerlendirilmesi konusunda yeterli bilgiye sahip olmayan kişilerin veriyi rahatlıkla değerlendirebilmesine olanak sunması ve ücretsiz olmasından dolayı ticari veya bilimsel yazılımın alınmasına gerek duyulmamasıdır. Bu çalışmada ANRK, ANTL, IZMI, SINP ve VAN1 istasyonlarına ait RINEX gözlemleri 1, 4, 8, 12, 16, 20 ve 24 saatlik dilimlere ayrılarak CSRS-PPP ve Trimble-RTX servislerine gönderilmiş ve elde edilen koordinatların doğruluk araştırması yapılmıştır. Çalışma sonucunda CSRS-PPP servisinden elde edilen konum verisinin referans veriye daha yakın olduğu anlaşılmıştır.
\end{abstract}

\section{Investigating Performance of Web-Based GNSS Software (CSRS-PPP, Trimble-RTX)}

\section{Keywords}

GNSS

CSRS-PPP

Trimble-RTX

\begin{abstract}
In recent years, online Global Navigation Satellite System (GNSS) evaluation services have been used as an important tool to obtain high accuracy in positioning. The most important feature of these systems is that GNSS data allows people who do not have any knowledge about evaluation to be able to evaluate the data easily and that they do not need commercial or scientific software because they are free of charge. In this study, RINEX observations of ANRK, ANTL, IZMI, SINP and VAN1 stations were divided into 1, 4, 8, 12, 16, 20 and 24-hour intervals and sent to CSRS-PPP and Trimble-RTX services and the accuracy of the coordinates was obtained. As a result of the study, it was understood that the positioning data obtained from CSRS-PPP service were closer to the reference data.
\end{abstract}

*(samed_inyurt@hotmail.com) ORCID ID 0000 - 0001 - 9339 - 7569 (mulukavak@harran.edu.tr) ORCID ID 0000 - 0003 - 2092 - 3075
INNYURT, S , ULUKAVAK, M . (2020). Web tabanlı GNSS Yazılımlarının (CSRS-PPP, TrimbleRTX) Performansının Araşırılması. Geomatik 5 (2), 120-126 . DOI: 10.29128 /geomatik.586990 Retrieved from https://dergipark.org.tr/tr/pub/geomatik/issue/52495/586990 


\section{GíRiş}

Uydu-bazlı konumlama yaparken mutlak ve bağll konum belirleme olmak üzere iki yöntem kullanılmaktadır. Fakat her iki yönteminde yüksek doğruluklu koordinat bilgisi elde etmede birtakım zorlukları vardır. Bu sebeple özellikle International GNSS Service (IGS) gibi kuruluşlar yüksek duyarlıklı yörünge bilgilerini üretmekte ve kullanıcılara ücretsiz olarak sunmasıyla konum belirleme üzerine çok sayıda yeni algoritma geliştirilmiştir. Bunlardan en fazla bilinen ve yoğun olarak kullanılanı Precise Point Positioning (PPP) tekniğidir. Sıfır fark alma (zero-difference) tekniğinin özel bir hali olan bu yöntem ile tek bir GNSS alıcısıyla koordinat bilgisi elde edilebilmektedir (Zumberge ve diğ., 1997; Kouba ve Héroux, 2001; Kouba, 2003; Gao ve diğ., 2005; Kouba, 2009; Cai ve Gao, 2007; Choy ve diğ., 2007). Son yıllarda PPP yöntemi kullanım kolaylığı, yüksek doğruluk elde etmesi, düşük maliyet sağladığından dolayı oldukça tercih edilmektedir. Bu yöntem uygulanırken herhangi bir referans GNSS alıcına veya Continuous Operating Reference Stations (CORS) ağına ihtiyaç duyulmamaktadır. Bu durum PPP çözümlemesini özellikle CORS ağının bulunmadığı bölgelerde yüksek doğruluk elde etmek için kullanılabilecek etkili yöntem olarak öne çıkarmaktadır. Bu sebeple 1990'lı yılların başından günümüze kadar pek çok araştırmacı PPP yöntemi üzerine çeşitli araştırmalar yapmıştır (Geng ve diğ., 2010; Huber ve diğ., 2010; Ocalan ve Alkan, 2012; Furones ve diğ., 2012; Elsobeiey ve El-Rabbany, 2013; Chen ve diğ., 2013; Liu ve diğ., 2013).

PPP tekniği herhangi bir şekilde sürekli gözlem yapan istasyona ihtiyaç duymamaktadır. Bertiger ve diğ., (2010) tarafından geliştirilen PPP yöntemi kullanış ve maliyet bakımından önemli avantajlar sağlamaktadır. Bazı üniversiteler ve araştırma merkezleri PPP yazılımları hazırlamıș ve online servisler aracılığıyla kullanıcıların ücretsiz kullanımına açmıştır. Bu servisler 24 saat kesintisiz bir şekilde kullanıma açıktır. Kullanıcı sadece elde ettiği GNSS gözlemini alıcı formatında veya RINEX formatında bu servislere yükleyerek çok kısa bir sürede ilgili noktaya ilişkin çözümlere ulaşır. Bu servisler ilgili veriye ilişkin çözümler yaparken yüklenilen gözlem dosyasına karşılık gelen hassas efemeris ve saat düzeltmeleri otomatik olarak indirilir ve çözümlerinde bunlar göz önüne alarak gerçekleştirilir. Nihai çözümler kullanıcılara e-mail aracılığıyla gönderilir. Elde edilen sonuçlar hassas konum ve kullanılan verinin kalitesine ilişkin çözümlere ek olarak, iyonosfer, troposfer ve alıcı saat hatası gibi sonuçları da kullanıcıya gönderir.

PPP tekniği kullanım olarak gerek bilimsel gerekse günlük hayatta giderek yaygınlaşmaktadır. GNSS hakkında yeterince bilgi sahibi olmayan fakat hassas konum bilgisine ihtiyaç duyan kişiler için bu teknik önemli avantaj sağlamaktadır. GhoddousiFard, and Dare'in (2006) yapmış olduğu çalışmada AUSPOS, SCOUT, OPUS, Auto-GIPSY ve CSRS-PPP servislerini kullanarak dünya genelinde 8 GNSS istasyonuna ilişkin çözümlemeler yapmış ve elde ettiği sonuçları karşılaştırmıştır. Guo (2015) yapmış olduğu çalışmada Ghoddousi-Fard, and Dare'in (2006) çalışmasında kullanılan gözlemleri APPS, CSRS-PPP, OPUS, AUSPOS, SCOUT ve Magic GNSS servislerini kullanarak analiz etmiş ve uyumlu sonuçlar elde etmiştir. Martín ve diğ, (2010) dört ayrı online servis kullanarak Bernese v5.0 yazılımını kullanarak elde ettiği koordinatları kesin koordinat olarak kabul edip koordinat farklarını incelemiştir. Çalışma sonucunda Bernese v5.0 yazılımından faydalanarak elde ettiği koordinatlar ile bu servislerden elde ettiği koordinatların birbirleriyle oldukça uyumlu olduğu tespit edilmiştir.

Bu çalışmada ANRK, ANTL, IZMI, SINP ve TVA2 istasyonlarına ait gözlemler 1, 4, 8, 12, 16, 20, 24 saatlik dilimler halinde CSRS-PPP ve Trimble-RTX GNSS veri değerlendirme servislerine gönderilmiş ve Harita Genel Müdürlüğü'nün (HGM) istasyonlara ilişkin sağlamış olduğu koordinatlar referans alınarak doğruluk analizi gerçekleștirilmiştir.

\section{MATERYAL ve METHOD}

\subsection{CSRS-PPP Yazılımı}

2003 yılında kullanıma açılan Canadian Spatial Reference System Precise Point Positioning (CSRSPPP), Natural Resources Canada (NRCAN) kuruluşu tarafindan kullanıcılara sunulmaktadır. Bu sistemi kullanabilmek için ilk olarak üyelik almak gerekmektedir. Üyelik işlemleri https://webapp.geod.nrcan.gc.ca/geod/accountcompte/login.php adresinden yapılmaktadır. Programın web ara yüzü Şekil 1'de gösterilmiştir.

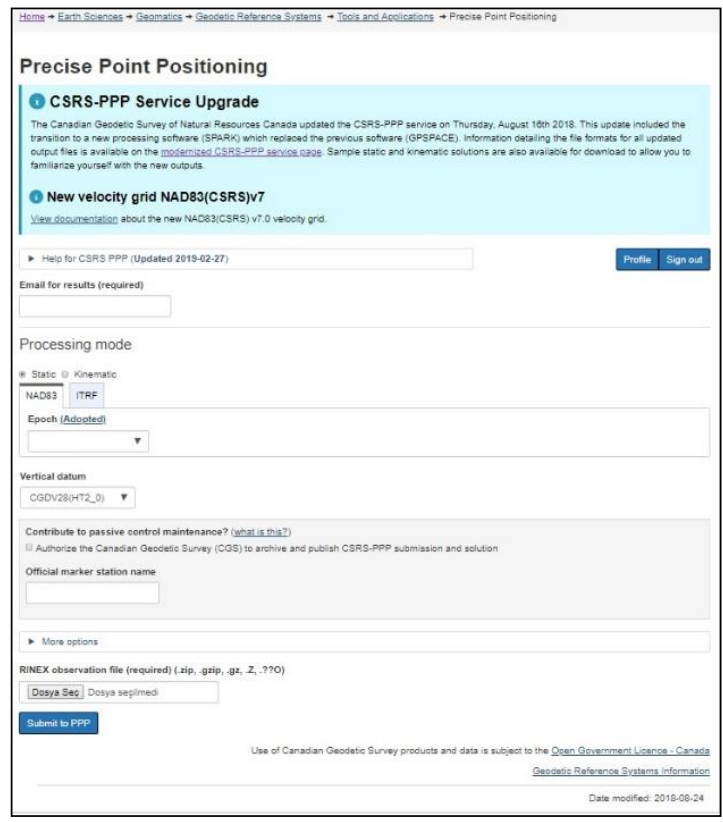

Şekil 1. CSRS-PPP Ara yüzü

Şekil 1'de görülen ara yüzden üyelik işlemlerini gerçekleştirdikten sonra ilgili gözlem dosyaları statik veya kinematik olarak değerlendirilebilmektedir. 


\subsection{Trimble-RTX Yazılımı}

GNSS sektöründe önemli bir yer tutan Trimble kurulușunun sağlamıș olduğu bu servis kullanıcılara ücretsiz olarak gözlem verilerini değerlendirme olanağ 1 sunmaktadır. https://www.trimblertx.com/UploadForm.aspx adresinden erişim sağlanan servis DAT, T01, T02, Quark, RINEX 2 ve RINEX 3 veri formatlarını değerlendirebilmektedir. Yazılım, 14 Mayıs 2011 tarihinden sonra sadece statik yöntemle yapılan gözlemleri değerlendirebilmektedir. BeiDou ve Galileo uydularından toplanan verileri de değerlendirebilen yazılıma ilişkin ara yüz Şekil 2'de verilmiștir.

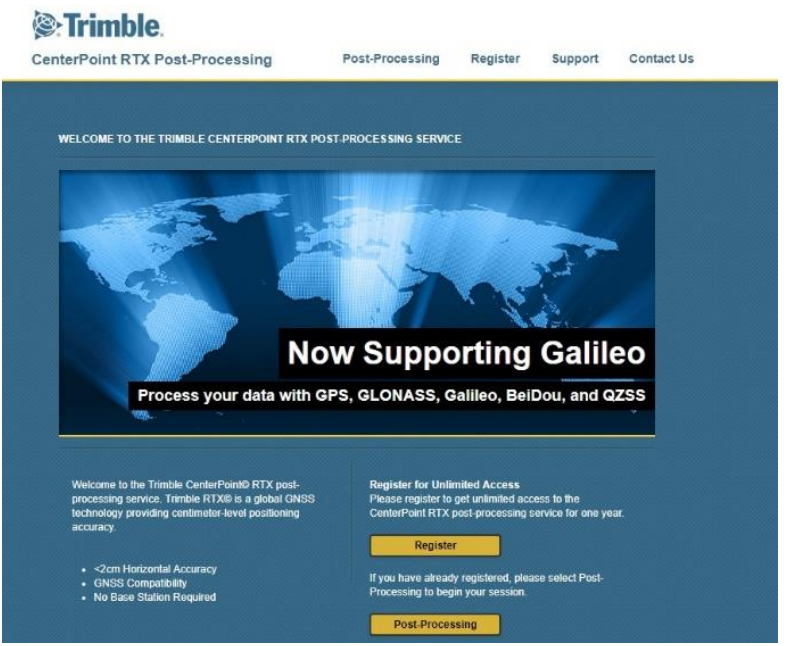

\section{BULGULAR ve TARTIŞMA}

Bu çalıșmada ANRK, ANTL, IZMI, SINP ve VAN1 TUSAGA-Aktif istasyonlarına ait 01.01.201401.02.2014 tarihleri arasındaki toplam 1 aylık RINEX formatındaki veriler $1,4,8,12,16,20$ VE 24 saatlik dilimlere ayrılmış ve yukarıda bahsedilen CSRS-PPP ve Trimble-RTX servislerine gönderilmiștir. Her iki siteden gelen sonuç dosyasında koordinata ilişkin veriler ITRF 2008 ölçüm epoğunda gelmektedir. Elde edilen sonuçların Harita Genel Müdürlüğü'nün (HGM) ilgili yll için üretmiş olduğu nihai koordinatlarla karşılaştırılabilmesi için servislerden elde edilen koordinatlar ITRF1996 sistemi 2005 epoğuna çevrilmiştir. Her iki yazılımdan elde edilen $\mathrm{X}, \mathrm{Y}$ ve $\mathrm{Z}$ koordinatları kullanılarak her istasyona ilişkin ortalama $\mathrm{X}, \mathrm{Y}$ ve $\mathrm{Z}$ koordinatları üretilmiş, daha sonra X, Y ve Z ortalama koordinatları ile HGM tarafindan yayınlanan koordinatlar arasındaki mutlak farklar irdelenmiştir (Şekil 3).

Şekil 2. Trimble-RTX Ara yüzü

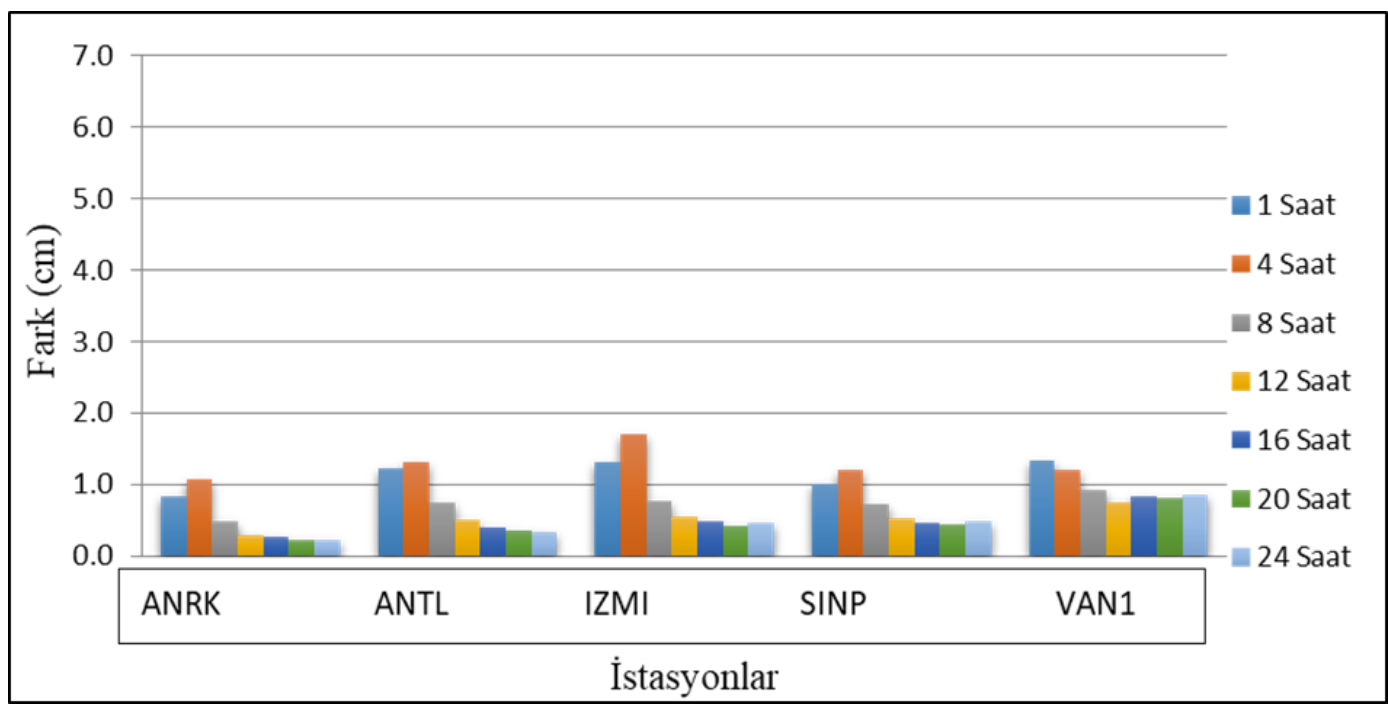

Şekil 3. ANRK, ANTL, IZMI, SINP, VAN1 istasyonlarının X Koordinat Farkları (CSRS-PPP) 
Şekil 3, ANRK, ANTL, IZMI, SINP ve VAN1 istasyonlarının 01.01.2014-01.02.2014 tarihleri arasında CSRS-PPP yazılımından elde edilen ortalama X koordinatı ile HGM'den elde edilen X koordinatları arasındaki mutlak farkı göstermektedir. Sonuçlara genel olarak bakıldığında gözlem süresi arttıkça farkın azaldığı görülmektedir. VAN1 istasyonu haricinde diğer tüm istasyonlarda 4 saatlik gözlem sonucu elde edilen koordinat ortalama farkının 1 saatlik gözlem sonucu elde edilen farktan daha düşük kaldığı tespit edilmiştir. Maksimum farkın IZMI istasyonunda 4 saatlik gözlem sonucu elde edilen ortalama X koordinatı ile HGM'nin yayınlamış olduğu X koordinatı arasında görülmektedir $(1.72 \mathrm{~cm})$.

Șekil 4, ANRK, ANTL, IZMI, SINP ve VAN1 istasyonlarının 01.01.2014-01.02.2014 tarihleri arasında CSRS-PPP yazılımı ile HGM'den elde edilen Y koordinatları arasındaki mutlak farkı göstermektedir.

Sonuçlara genel olarak bakıldığında gözlem süresi arttıkça farkın azaldığı görülmektedir. Maksimum fark VAN1 istasyonunda 1 saatlik gözlem sonucu elde edildiği görülmektedir $(1.63 \mathrm{~cm})$.

Șekil 5 irdelendiğinde X bileșeninde olduğu gibi 4 saatlik gözlemler sonucu elde edilen $\mathrm{Z}$ koordinat farklarının 1 saatlik gözlem sonucu elde edilen farklardan daha fazla olduğu tespit edilmiştir (VAN1 istasyonu hariç). Bu bileşende maksimum fark IZMI istasyonunda 4 saatlik gözlem sonucu $1.83 \mathrm{~cm}$ olarak tespit edilmiştir.

Trimble-RTX yazılımından elde edilen X koordinatlarından üretilen mutlak $\mathrm{X}$ değerleri ile HGM'den elde edilen X koordinatına ilişkin farklar Şekil 6'da verilmiştir.

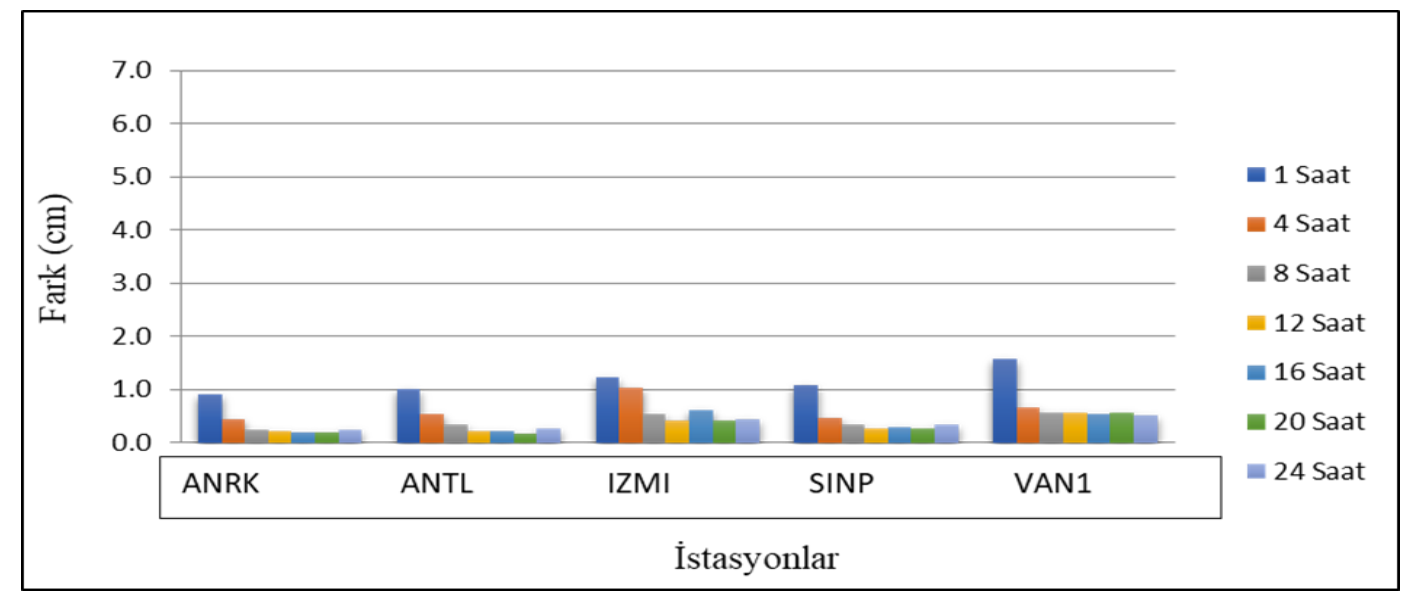

Şekil 4. ANRK, ANTL, IZMI, SINP, VAN1 istasyonlarının Y Koordinat Farkları (CSRS-PPP)

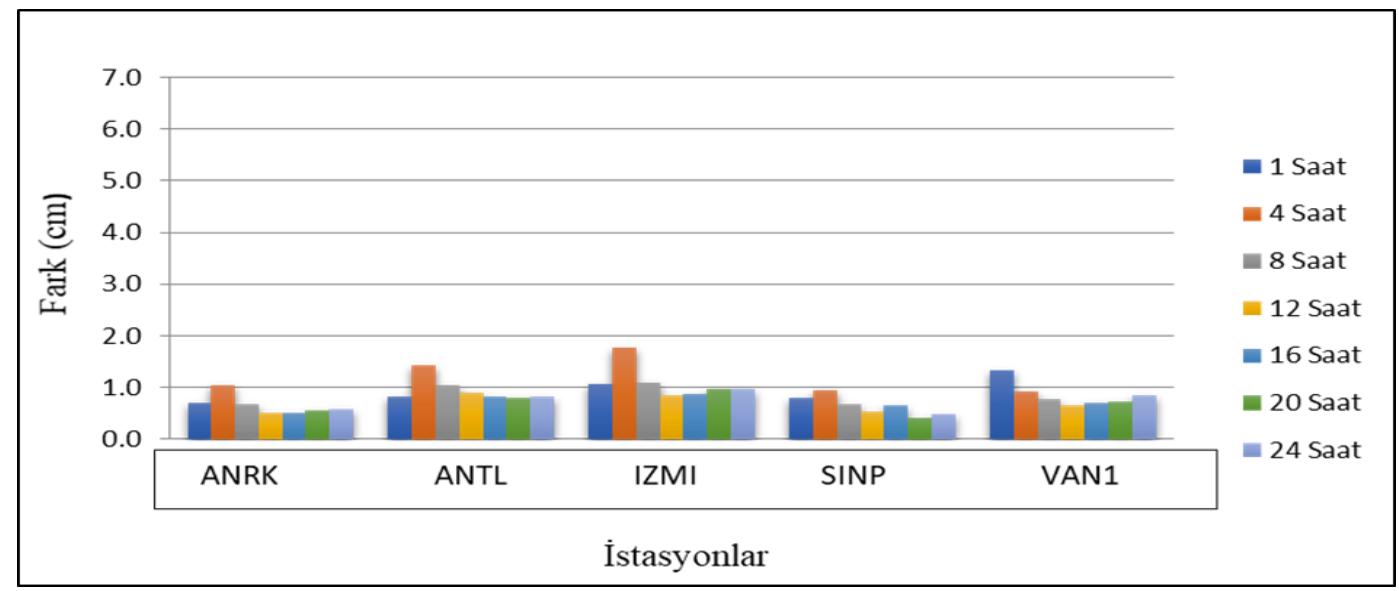

Şekil 5. ANRK, ANTL, IZMI, SINP, VAN1 istasyonlarının Z koordinat farkları (CSRS-PPP) 


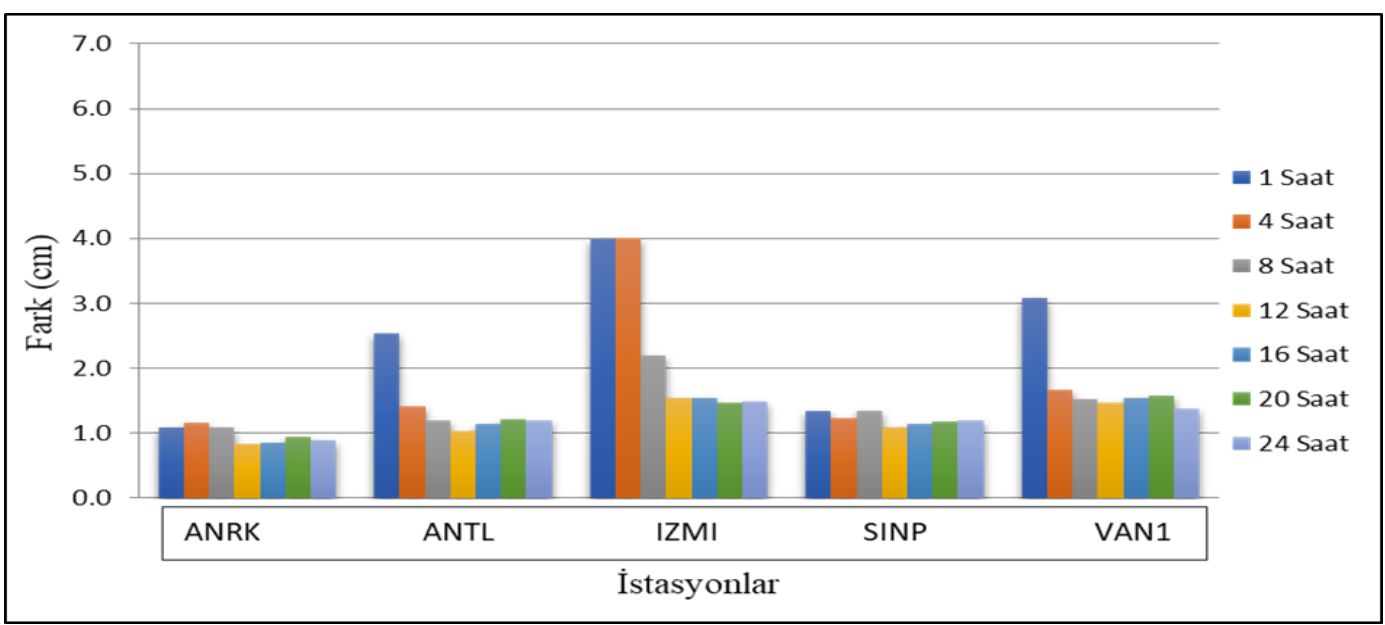

Şekil 6. ANRK, ANTL, IZMI, SINP, VAN1 istasyonlarının X koordinat farkları (CSRS-PPP)

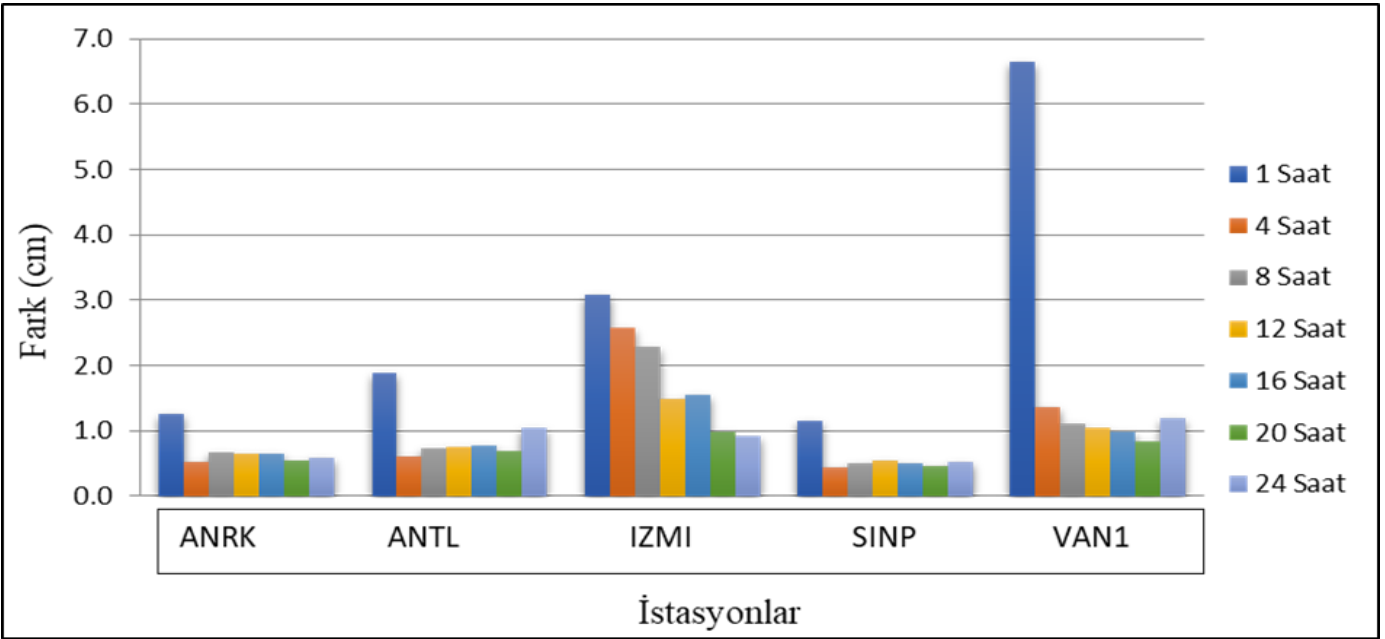

Şekil 7. ANRK, ANTL, IZMI, SINP, VAN1 istasyonlarının Y Koordinat Farkları (Trimble RTX)

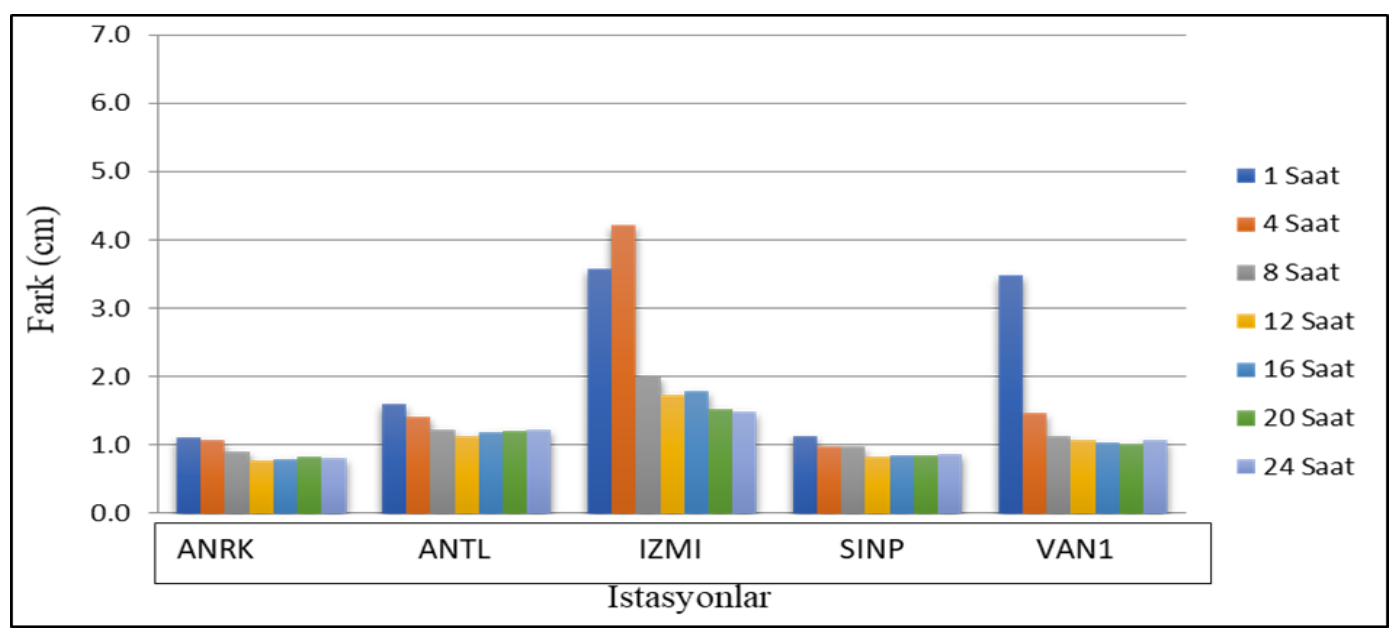

Şekil 8. ANRK, ANTL, IZMI, SINP, VAN1 istasyonlarının Z Koordinat Farkları (Trimble RTX) 
Şekil 6'da ANRK, ANTL, IZMI, SINP ve VAN1 istasyonlarının Trimble RTX yazılımından elde edilmiș X koordinatlarından faydalanılarak elde edilen ortalama $X$ koordinatları ile HGM'nin yayınlamış olduğu X koordinatları arasındaki mutlak fark gösterilmektedir. Sonuçlar irdelendiğinde maksimum farkın IZMI istasyonunda 1 ve 4 saatlik gözlem sonucunda elde edildiği görülmektedir (4 $\mathrm{cm})$.

Şekil 7'de analiz yapılan istasyonlara ilişkin ortalama Y koordinatı ile HGM tarafından yayınlanan Y koordinatı arasındaki mutlak farklar gösterilmektedir. Maksimum fark $(6.77 \mathrm{~cm}) 1$ saatlik gözlem sonucu VAN1 istasyonunda gözlemlenmiştir. Sonuçlar irdelendiğinde ANRK, IZMI, ANTL, SINP istasyonlarında tüm gözlemler sonucu elde edilen farkların birbirine çok yakın olduğu görülmüștür. VAN1 istasyonunda 1 saatlik gözlem sonucu elde edilen fark haricinde diğer tüm sonuçların diğer istasyonlarda olduğu farkın $\mathrm{mm}$ mertebesinde olduğu görülmektedir.

Şekil 8'de Z koordinatına ilişkin Trimble RTX yazılımından elde edilen farklar gösterilmiştir. Yapılan analiz sonuçlarına bakıldığında maksimum farkın $4.2 \mathrm{~cm}$ ile IZMI istasyonuna ait olduğu görülmektedir. Z koordinatına ilișkin elde edilen çözümlerde ANRK, ANTL, SINP istasyonlarında farkların mm seviyesinde olduğu, IZMI istasyonunda 1 saatlik gözlemden elde edilen farkların 4 saatlik gözleme göre daha düșük kaldığı tespit edilmiștir. Diğer yandan VAN1 istasyonunda 1 saatlik gözlem sonucu hariç olmak üzere tüm gözlemlerden elde edilen sonuçların birbirine oldukça yakın olduğu görülmektedir.

\section{SONUÇLAR ve ÖNERILER}

Bu çalışmada ANRK, ANTL, IZMI, SINP ve VAN1 TUSAGA-Aktif istasyonlarına ait 01.01.201401.02.2014 tarihleri arasındaki gözlem dosyaları 1, 4, 8, 12, 16, 20 ve 24 saatlik dilimlere ayrılmış, CSRSPPP ve Trimble-RTX servislerine gönderilmiştir. İki servisten elde edilen her güne ilișkin ITRF 2008 gözlem epoğunda elde edilen koordinatların ortalaması alınmış ve kartezyen koordinat sistemine dönüştürülmüștür. Bu servislerden elde edilen $\mathrm{X}, \mathrm{Y}$ ve Z koordinatları ile aynı istasyonlara ait HGM'nin yayınlamış olduğu kartezyen koordinatlar arasındaki farklar incelenmiştir. CSRS-PPP yazılımından elde edilen sonuçlara bakıldığında X koordinatında maksimum farkın $1.72 \mathrm{~cm}$ ile IZMI istasyonunda, Y koordinatında maksimum farkın VAN1 istasyonunda $1.63 \mathrm{~cm}, \mathrm{Z}$ koordinatında ise maksimum farkın $1.83 \mathrm{~cm}$ ile IZMI istasyonunda olduğu görülmektedir. Trimble-RTX yazılımı kullanılarak yapılan analiz sonuçlarına bakıldığında $X$ koordinatında maksimum farkın IZMI istasyonunda $4 \mathrm{~cm}$, Y koordinatında $6.77 \mathrm{~cm}$ ile VAN1 istasyonunda, $\mathrm{Z}$ koordinatında $4.2 \mathrm{~cm}$ ile IZMI istasyonunda olduğu görülmektedir. Her iki yazılım birbiri ile kıyaslandığında genel olarak CSRS-PPP yazılımının HGM'nin çözümlerine daha yakın sonuçlar verdiği anlaşılmaktadır.

\section{KAYNAKÇA}

Bertiger, W., Desai, S. D., Haines, B., Harvey, N., Moore, A. W., Owen, S., Weiss, J. P. (2010). Single receiver phase ambiguity resolution with GPS data. Journal of Geodesy, 84(5), 327-337.

Cai, C., and Gao, Y. (2007). Precise point positioning using combined GPS and GLONASS observations. Positioning, 1 (11).

Chen, J., Li, H., Wu, B., Zhang, Y., Wang, J., and Hu, C. (2013). Performance of real-time precise point positioning. Marine Geodesy, 36(1), 98-108.

Choy, S. L., Zhang, K., Silcock, D., and Wu, F. (2007). Precise Point Positioning-a case study in Australia. In Proceedings of spatial sciences institute international conference (SSC2007), Tasmania, pp. 192-202.

Elsobeiey, M., and El-Rabbany, A. (2013). Efficient between-satellite single-difference precise point positioning model. Journal of Surveying Engineering, 140(2).

Furones, M., Esteban, Á., Anquela Julián A. B., Berné Valero J. L., and Sanmartín, M. (2012). Kinematic GNSS-PPP results from various software packages and raw data configurations. In Scientific Research and Essays (Vol. 7, No. 3, pp. 419-431). Academic Journals.

Gao, Y., Wojciechowski, A., and Chen, K. (2005). Airborne kinematic positioning using precise point positioning methodology. Geomatica, 59(1), 29-36.

Geng, J., Teferle, F. N., Meng, X., and Dodson, A. H. (2010). Kinematic precise point positioning at remote marine platforms. GPS solutions, 14(4), 343-350.

Ghoddousi-Fard, R., and Dare, P. (2006). Online GPS processing services: an initial study. GPS solutions, 10(1), 12-20.

Guo, Q. (2015). Precision comparison and analysis of four online free PPP services in static positioning and tropospheric delay estimation. GPS solutions, 19(4), 537-544.

Huber, K., Heuberger, F., Abart, C., Karabatic, A., and Weber, R. (2010). PPP: Precise Point Positioning-constraints and opportunities. 
Kouba, J., and Héroux, P. (2001). Precise point positioning using IGS orbit and clock products. GPS solutions, 5(2), 12-28.

Kouba, J. (2003). Measuring seismic waves induced by large earthquakes with GPS. Studia Geophysica et Geodaetica, 47(4), 741-755.

Kouba, J. (2009). A guide to using International GNSS Service (IGS) products.

Liu, Z., Ji S., Chen, W., and Ding, X. (2013). New fast precise kinematic surveying method using a single dual-frequency GPS receiver. Journal of Surveying Engineering, 139(1), 19-33.

Martín, A., Anquela, A. B., Capilla, R., and Berné, J. L. (2010). PPP technique analysis based on time convergence, repeatability, IGS products, different software processing, and GPS+ GLONASS constellation. Journal of Surveying Engineering, 137(3), 99-108.

Ocalan, T., and Alkan, R. M. (2012). Performance Analysis of Web-Based Online Precise Point Positioning (PPP) Services for Marine Applications. In The 14th IAIN Congress.

Zumberge, J. F., Heflin, M. B., Jefferson, D. C., Watkins, M. M., and Webb, F. H. (1997). Precise point positioning for the efficient and robust analysis of GPS data from large networks. Journal of Geophysical Research: Solid Earth, 102(B3), 5005-5017. 